\title{
A Cross-sectional Study Using Wireless Electrocardiogram to Investigate Physical Workload of Wheelchair Control in Real World Environments
}

\author{
Shawn Joshi ${ }^{1-5}$, Roxana Ramirez Herrera ${ }^{6}$, Daniella Nicole Springett ${ }^{3-5}$, Benjamin \\ David Weedon ${ }^{3-5}$, Dafne Zuleima Morgado Ramirez ${ }^{6,7}$, Catherine Holloway ${ }^{6,7}$, \\ Hasan Ayaz ${ }^{1,9,10}$, Helen Dawes ${ }^{3-5}$ \\ ${ }^{1}$ School of Biomedical Engineering, Science \& Health Systems, Drexel University, \\ Philadelphia PA, USA \\ ${ }^{2}$ College of Medicine, Drexel University, Philadelphia PA, USA \\ ${ }^{3}$ Movement Science Group, Oxford Brookes University, Oxford, UK \\ ${ }^{4}$ Oxford Institute of Nursing, Midwifery, and Allied Health Research, Oxford, UK \\ ${ }^{5}$ Nuffield Department of Clinical Neurosciences, Oxford University, Oxford, UK \\ ${ }^{6}$ UCL Interaction Centre, University College London, UK \\ ${ }^{7}$ Global Disability Innovation Hub, London, UK \\ ${ }^{8}$ Department of Family and Community Health, University of Pennsylvania, \\ Philadelphia PA, USA \\ ${ }^{9}$ Center for Injury Research and Prevention, Children's Hospital of Philadelphia, \\ Phialdelphia, PA, USA \\ ${ }^{9}$ Drexel Business Solution Institute, Drexel University, Philadelphia, PA, USA \\ \{sj633, ayaz\}drexel.edu, hdawes@brookes.ac.uk
}

\begin{abstract}
The wheelchair is a key invention that provides individuals with limitations in mobility increased independence and participation in society. However, wheelchair control is a complicated motor task that increases physical and mental workload. New wheelchair interfaces, including power-assisted devices can further enable users by reducing the required effort especially in more demanding environments. The protocol engaged novice wheelchair users to push a wheelchair with and without power assist in a simple and complex environment using wireless Electrocardiogram (ECG) to approximate heart rate (HR). Results indicated that HR determined from ECG data, decreased with use of the power-assist. The use of power-assist however did reduce behavioral performance, particularly within obstacles that required more control.
\end{abstract}

Keywords: Wheelchair · Power-Assist $\cdot$ Heart Rate $\cdot$ Wireless $\cdot$ Real-World Cognitive Workload · Disability

\section{Introduction}

The wheelchair is a tool for equality for individuals with limitations in mobility; it increases independence and opportunities to actively engage in their environment [1, 2]. Typical manual wheelchair propulsion can lead to a variety of negative health outcomes. Wheelchair users (WU) are prone to upper arm injuries related to continuous or excessive use, including damage to rotator cuff muscles $-42-66 \%$ of WU often report shoulder pain [3-5] and may suffer from bilateral carpal tunnel syndrome [6]. 
Reduced mobility can lead to a more sedentary lifestyle often reducing individual physical capacity for many WU [7, 8].

This is particularly problematic, as independent manual wheelchair propulsion requires adept physical capacity and cardiorespiratory fitness [9, 10]. Many musculoskeletal problems manual WU face can be prevented by reducing the use of the wheelchair (however this is wholly impractical as it would limit equality/autonomy), or altering factors related to reducing the physical load (demands of the environment) [11, 12], or increasing power (human characteristics) [13]. Electric remove the need to selfpropel and therefore reduce strain injuries, while reducing metabolic demand to allow further travel and in more variable locations [14]. However they encourage an even less physically active lifestyle, predisposing users to long term health problems related to inactivity (obesity, cardiovascular disease, etc.) $[15,16]$.

Power Assisted Devices (PADs) are new generation mobility interfaces, that offer a middle ground solution to the problems of both manual and electric wheelchairs. They can allow users to reduce physical strain, but not at the cost of removing all the cardiovascular (CV) beneficial physical activity [13]. They are propelled in the same manner as a manual wheelchair but are fitted with small electric motors (either in the wheels or behind the wheelchair) to augment the user's physical power and allow for the social, and mobile benefits of an electric wheelchair while partially retaining the exercise component from a manual wheelchair. While PADs have intrinsic design problems, they are being increasingly considered among manual WU [17].

Wheelchair control is a complicated motor task that increases both the cognitive (or mental) and physical workload of an individual [18]. Cognitive workload (CW) refers to the limited information processing capacity of the brain demanded by a task or environment [19]. When environmental demands increase, subsequent increases in $\mathrm{CW}$ are generated. However if environmental demands exceed this capacity for information processing, task performance inevitably decreases [20]. Accidents or errors are a result of decreased or poor task performance [21]. Measuring CW is complex as it represents the interplay between the environmental demands (input), human characteristics (capacities), and task performance (output) on the operator [22, 23]. The association between $\mathrm{CW}$ and physical workload is an essential component of physical neuroergonomics, the study of the brain in relation to the control and design of physical tasks incorporating evaluations of brain and body measurements in natural environments as opposed to artificial laboratory settings and simplified tasks [24-32].

Understanding the factors in reducing/optimizing cognitive and physical workload in in order to improve task performance is important, particularly in the context of operating complex machinery such as manual wheelchairs. Excessive workload can lead to serious injuries, increased economic burden, and other maladies to and from the user $[33,34]$ and can further impact mobility, resulting in activity restriction, affecting social participation, health and wellbeing and quality of life [35]. Physical workload can be measured by a variety of mechanisms, however one of the more common, practical and valid measures includes heart rate (HR) [36].

In the United States alone, there are 3.6 million active WU above the age of 15 , and due to our aging population, there are an additional 2 million new WU every year [37]. Therefore, it is imperative that newer generation wheelchair designs, such as PAD's, consider both physical and mental effort implications to optimize control ergonomics to improve safety and better community engagement. The objective of this 
study was to understand the interplay of expertise, environment, and interface during real-world wheelchair control. Therefore, this paper set out to evaluate the cognitive and physical workload as measured by behavioral task performance and HR for novice WU during manual and power-assisted wheelchair propulsion in both simple and complex environments.

\section{$2 \quad$ Methods}

30 novice participants ( 12 males) were recruited aged $31.8 \pm 9 y$ yrs. Only those physically able to propel a manual wheelchair for an extended period of time, and without cognitive disability or recent physical injury were recruited. Each participant completed a Physical Activity Readiness Questionnaire (PAR-Q) [38], and had biometric measurements of height, weight, age, skin color, hair color/type, grip strength (left and right), blood pressure, maximal speeds, seat height, and arm lengths. All participants also reported having normal or corrected-to-normal vision and were self-described to be able to control a wheelchair for up to one hour, including difficult terrain.

The study was conducted at the Oxford Brookes Sports Hall located in Oxford, UK with approval obtained from the University Research Ethics Committee with reference number UCLIC/1617/024/StaffHolloway/Herrera between 12/17 and 09/18.

\subsection{Measurments and Devices}

All participants used the manual wheelchair frame (QUICKIE LIFE R) weighing $10.5 \mathrm{~kg}$ to traverse two environments detailed below. The wheelchair had a seat width of $45 \mathrm{~cm}$ and fitted with the M24 Alber Twion (Alber GmbH, Albstadt, Germany) power assist wheels (additional 6kg each). The power assist was set in the ECO mode to allow for a maximal propulsion speed of $10 \mathrm{~km} / \mathrm{hr}$. Participants wore a portable ECG sensor known as the EcgMove 3 (Movisens GmbH, Karlsruhe, Germany) across the chess below T4. Experiments were video recorded using a GoPro Hero Action Digital.

The behavioral performance during the experiment was manually recorded by two research assistants (reduced inter-rater variability) and retrospectively corrected when reviewing the video of each experiment. Total number of errors per obstacle was recorded and converted into percentages based on the maximum error count recorded by all participants. Obstacle percentage scores were averaged to give a total performance percentage (each obstacle given the equal weight/importance), where higher scores indicated better performance/fewer errors.

\subsection{Environmental Design}

Two environments (simple and complex) were created. The simple environment (flat terrain and free of obstacles) formed the outer rectangle of $13 \mathrm{~m} \times 14 \mathrm{~m}$ for a total propulsion distance of $54 \mathrm{~m}$, and the complex environment (four separate obstacles) nested within a $36 \mathrm{~m}$ inner square. Each obstacle was approximately $7 \mathrm{~m}$, with $1 \mathrm{~m}$ of free space between the start and end of the obstacle to allow for clearance and preparation for the next obstacle. All obstacles were designed to mimic common conditions WU encounter, two required more power (rough terrain and incline slope), and two of which required more motor planning (cones and side slopes). The overall 
design of the environments and order of the obstacles are depicted in Figure 1, and further described below. The environments were set in the Oxford Brookes Sports Hall and guiding lines were provided for participants to follow.

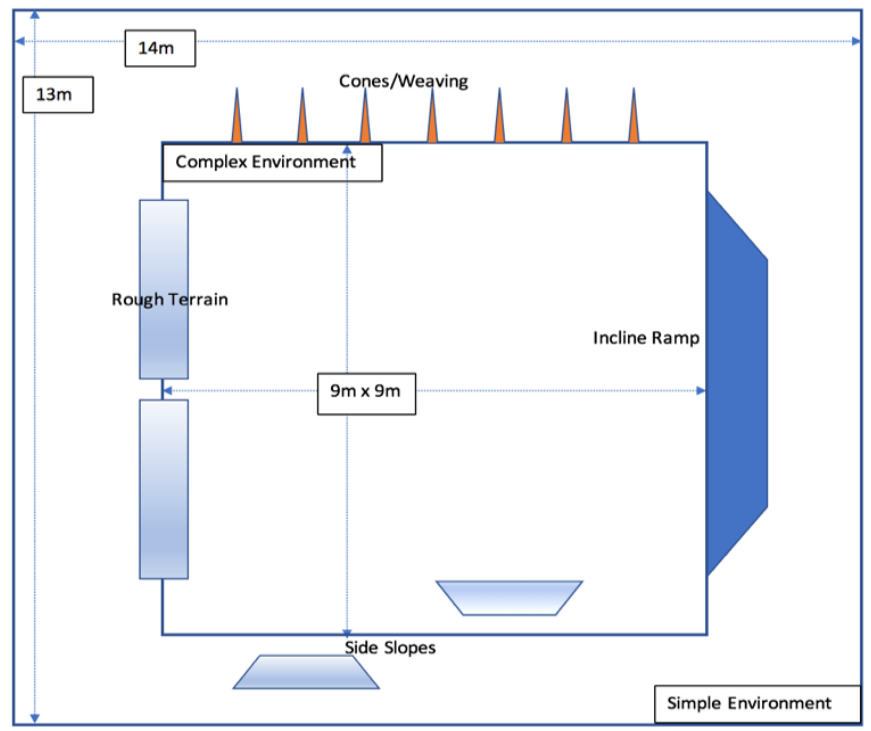

Figure 1. Sketch of outer simple and nested inner complex (with 4 obstacles) environments.

The obstacle labelled as "Rough Terrain" in Figure 1 mimicked a high friction environment requiring more power from the user, and was created using foam noodles, a common material used in obstacle designs for children with disabilities [39]. As specified by the wheelchair manufacturer manual, the height of the rough terrain was roughly set at $3 \mathrm{~cm}$ repeated every $3-5 \mathrm{~cm}$, under the $5 \mathrm{~cm}$ safety limit for the caster wheels and at a total width of $80 \mathrm{~cm}$. Errors while traversing rough terrain including shifting off the obstacle path, hesitating or abruptly stopping while traversing.

The incline ramp also required more power, created from $1.8 \mathrm{~cm}$ thick plywood and set $1 \mathrm{~m}$ wide with safety barriers to prevent participants from falling off the ramp. The incline ramp was set to American Disability Association (ADA) 2010 guidelines [37] of having a maximum of a $5^{\circ}$ gradient at a straight on approach. The ramp was $3 \mathrm{~m}$ long, climbing to a horizontal surface at a height of $26 \mathrm{~cm}$ and $50 \mathrm{~cm}$ in length that continued to an additional $3 \mathrm{~m}$ decline at the same $5^{\circ}$ gradient to reach back to the flat path. Physical errors while traversing the incline ramp included hitting the boundary lip of the ramp as well as hesitating/stopping during the entire obstacle.

Cones/weaving required more upper limb coordination and control. Cones were set on the guiding line of the path at $92 \mathrm{~cm}$ apart (cone edge to cone edge) to mimic ADA guidelines [37] of acceptable wheelchair accessible door width. The start and final cone were set $1.1 \mathrm{~m}$ from the ends of the length. The participants were asked to approach the first cone from the outside, and weave back and forth until reaching the end. Errors while traversing the cones included hitting or ignoring a cone.

The side slopes also required more upper limb coordination and control. Each slope was $2.4 \mathrm{~m}$ in length and set at a $10^{\circ}$ gradient to a maximum of $20 \mathrm{~cm}$, a height tested to 
be safely balanced and not lead to tipping over. They were set $1.5 \mathrm{~m}$ from the ends of the length, and $70 \mathrm{~cm}$ away from the path at a parallel angle. The participants were instructed to approach the first side slope at an angle using one wheel on the side slope while keeping the other wheel along the flat path. The participants were requested to exit the side slope and approach the $2^{\text {nd }}$ side slope with the other wheel while maintaining the remaining wheel on the flat path. Errors while traversing the side slopes included hesitations and not maintaining a level height on the slope.

\subsection{Experimental Setup}

All circuits were completed in clockwise and counterclockwise directions alternating every 4 circuits during the experiment. This setup was designed to prevent fatigue. All circuits were completed in a pseudorandomized predetermined order per participant to reduce a repetitive learning effect. Ultimately, participants completed 16 circuits - 4 in a simple environment (no obstacles) without power assistance, 4 in a simple environment with power assistance, 4 in a complex environment (with obstacles) without power assistance, and 4 in a complex environment with power assistance.

To standardize the experiment participants were fixed at self-paced speeds. Each participant completed their first circuit through the complex environment without power assistance, at self-selected speeds (encouraged to make the fewest errors), to control for inter-individual differences in fitness, and recorded their first circuit completion time. All remaining circuits were attempted to be completed within that specific time ( \pm 5 seconds) regardless of interface by using a research assistant who walked beside the participant at that designated pace. The \pm 5 seconds accounted for fatigue and learning. Participants were given rests before the start of each circuit (3050 s) to allow for a more stable physiological baseline of HR and other measures. Total times to complete each circuit were recorded.

\subsection{Statistical Analysis}

Statistical analysis of behavioral performance (percentages based on errors) and HR information during the experimental procedure employed the use of linear mixed modeling implemented in NCSS (NCSS, LLC. Kaysville, Utah, USA). Linear mixedeffects estimates were computed with restricted maximum likelihood.

\section{Results}

All 30 novice behavioral performance data revealed a significant effect for the type of interface (manual or power-assist) $\left(\mathrm{F}_{1,209}=38.3877, \mathrm{p}<0.001\right)$ depicted in Figure 2. Use of the power-assist interface led to an overall decreased performance of $5.6179 \%$ for the complex environment. Post-hoc analysis with Bonferroni adjustment revealed that the power-assist interface significantly decreased performance for only 2 of the 4 obstacles (Figure 3 ) - 10.6667\% performance reduction for navigating the cones $\left(\mathrm{F}_{1,209}\right.$ $=32.1538, \mathrm{p}<0.001)$, and $9.1667 \%$ reduction for navigating the side slopes $\left(\mathrm{F}_{1,209}=\right.$ $15.3360, \mathrm{p}<0.001)$, but no significant difference in the obstacles that required more power, the rough terrain or the incline ramp. 


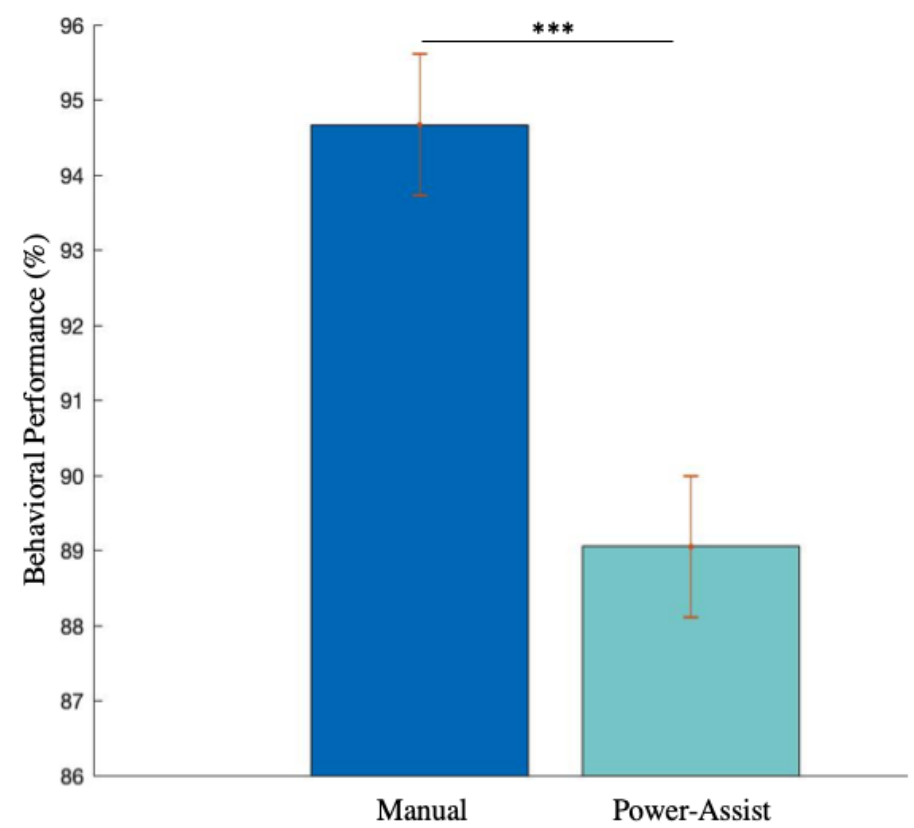

Figure 2. Overall behavioral performance comparison between the manual and powerassist interface for the entire complex environment. $(* * * p<0.001)$

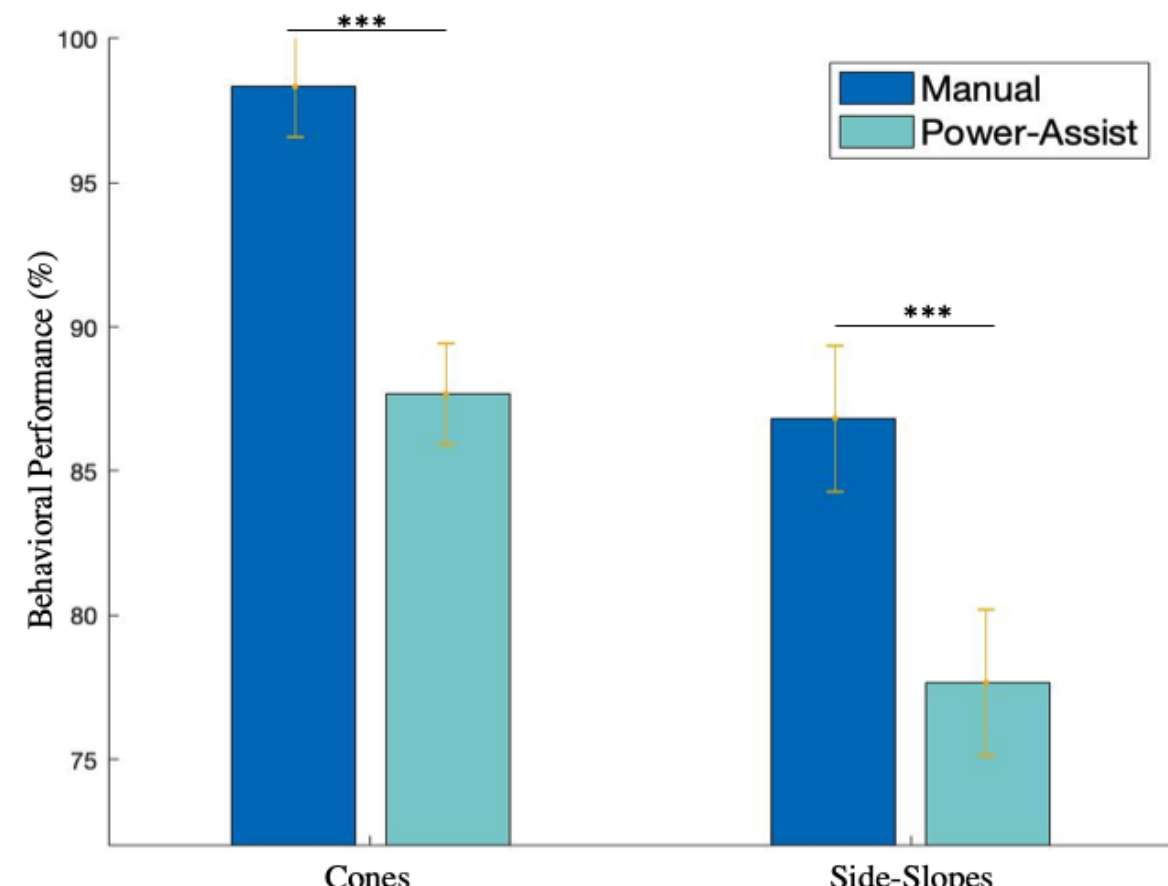

Figure 3. Behavioral performance comparison of the Cones and the Side Slopes between the manual and power-assist interface. $(* * * p<0.001)$ 
ECG data of 23 out of 30 novice participants was processed using a customized MATLAB script to calculate mean HR per circuit. Post-hoc analysis with Bonferroni adjustment revealed that HR of novice users decreased by $4.4767 \mathrm{bpm}$ with the use of power-assist $\left(\mathrm{F}_{1,328}=13.0175, \mathrm{p}<0.001\right)$. There was no significant difference in heart rate information between the different environments and there was no significant interaction between the interfaces and the environment.

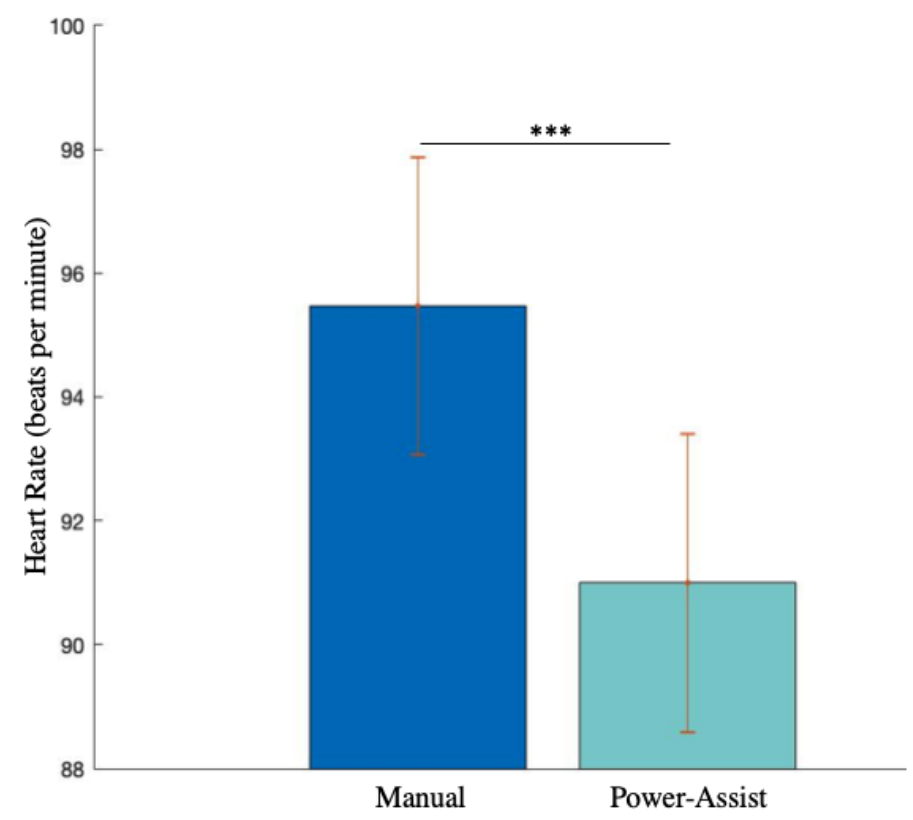

Figure 4. Heart Rate comparison of the interface. $(* * * p<0.001)$

\section{Discussion}

This study set out to explore the concept of PADs in physical and cognitive workload in realistic settings. As newer assistive device interfaces reach users with disabilities, it becomes paramount to begin understanding how these devices affect mental and physical workload within typical environments. We designed tasks that mimicked some of everyday situations WU would encounter. The results of this study observed that HR decreased with the use of power-assist for people learning to use wheelchairs, however at the cost of behavioral performance, particularly with particularly significant increases in errors for obstacles that require more skill and control. Therefore, this methodology along with HR measurements may aid in characterizing physical workload impact of power-assist interfaces. It appeared that new users were able to take advantage of the power assist to reduce physical workload as measured by HR while maintaining performance in tasks that require more power, but decreased performance in obstacles needing more control. 


\subsection{Environment}

Each subject completed 8 simple, and 8 complex circuits, where the complex circuits were predicted to increase the physical workload. However, HR was not a reliable predictor for determining environmental difficulty. Furthermore, no interactions between the interface and environment were found. WU develop skills over time to negotiate inaccessible environments [40, 41], however increased environmental difficulty did not induce measurable changes in HR as expected from the literature [42]. Environmental complexity [43], as related to task demands, are directly correlated with increased CW which has been determined via evaluation of task performance [44] and cardiac responses [45], however this was not the case for measuring HR within this study.

\subsection{Interface}

PAD's are designed to augment the physical power of the user, to reduce metabolic effort and allow the user to expend less energy than typical manual propulsion. This design intention was indeed reflected with a statistically significant, yet small decrease in HR of 4.48bpm. Several studies have explored exercise ergonomics, and even some in wheelchair ergonomics with similar findings [46], however none to our knowledge have looked at $\mathrm{CV}$ responses to the newer generation wheelchair interface of powerassistance. Champagne et. al reported similar cardiorespiratory reductions with the use of mobility assistance dogs during a natural environment [47]. This reduction in physical workload may be an important factor in allowing new users to further engage in their communities for increased social participation and equality even within nonoptimal environments.

Behavioral performance did however marginally decrease with the use of the power-assistance. This may indicate that as a new interface, power assistance may lead to more accidents specifically for those new to wheelchair control. This performance reduction/increased error rate was particularly true for obstacles that required more fine control and skill (weaving through cones and balancing on the side slopes). However, PAD's may not impact quality of control in environments that require more power (like high friction/rough environments and steep incline ramps). This may lead to more informed, customized decision making on the part of the user, to determine what types of environments they may face more regularly and whether PADs are optimal for their use.

\section{Conclusion}

In summary, HR is a reliable measurement for assessing the potential for physical workload reduction for power-assist devices/or new mobility interfaces for new users. Power-assistance is an important factor in reducing physical workload for people learning to use wheelchairs, but perhaps at the cost of increasing minor accidents/errors. Portable non-invasive ECG is a safe and reliable measure that can be used in any simple, or physically demanding environment. Measuring HR variability may be an important factor in future studies, along with more robust measures for behavioral performance including smoothness of control. Ultimately, portable physiological measures of WU 
in natural environments can provide more insight for personalization of mobility devices or improved guided skill training in wheelchair control.

Acknowledgments. We would like to thank Jamie Whitty and Joel Chappell of the School of Architecture from Oxford Brookes University, for constructing our ramps, Ian Allen, the Oxford Brookes sports booking coordinator, for helping us with numerous appointments, and our research assistants Cyrus Goodger, Jessica Andrich, and JoJo Dawes. This research was funded through the Adaptive Assistive Rehabilitative Technologies - Beyond the Clinic grant by the Engineering and Physical Sciences Research Council (EP/M025543/1). SJ is additionally supported by the Fulbright US-UK Commission. HD is supported by the Elizabeth Casson Trust and received support from the NIHR Oxford health Biomedical Research Centre. Additional support provided by CONACYT (National Council of Science and Technology in Mexico).

\section{References}

1. McClure, L.a., Boninger, M.L., Oyster, M.L., Williams, S., Houlihan, B., Lieberman, J.a., Cooper, R.a.: Wheelchair repairs, breakdown, and adverse consequences for people with traumatic spinal cord injury. Archives of physical medicine and rehabilitation 90, 2034-2038 (2009)

2. Smith, C., McCreadie, M., Unsworth, J., Wickings, H.I., Harrison, A.: Patient satisfaction: an indicator of quality in disablement services centres. Quality in Health CareQuality in Health Care 44, 31-3631 (1995)

3. Dalyan, M., Cardenas, D.D., Gerard, B.: Upper extremity pain after spinal cord injury. Spinal Cord 37, 191-195 (1999)

4. Fullerton, H.D., Borckardt, J.J., Alfano, A.P.: Shoulder Pain: A Comparison of Wheelchair Athletes and Nonathletic Wheelchair Users. Medicine \& Science in Sports \& Ecercise 35, 1958-1961 (2003)

5. Holloway, C.S., Symonds, A., Suzuki, T., Gall, A., Smitham, P., Taylor, S.: Linking wheelchair kinetics to glenohumeral joint demand during everyday accessibility activities. In: 2015 37th Annual International Conference of the IEEE Engineering in Medicine and Biology Society (EMBC), pp. 2478-2481. (2015)

6. Asheghan, M., Hollisaz, M.T., Taheri, T., Kazemi, H., Aghda, A.K.: The prevalence of carpal tunnel syndrome among long-term manual wheelchair users with spinal cord injury: A cross-sectional study. The Journal of Spinal Cord Medicine 39, 265-271 (2016)

7. Tawashy, A.E., Eng, J.J., Krassioukov, A.V., Miller, W.C., Sproule, S.: Aerobic Exercise During Early Rehabilitation for Cervical Spinal Cord Injury. Physical Therapy 90, 427-437 (2010)

8. Van Den Berg-Emons, R.J., Bussmann, J.B., Haisma, J.A., Sluis, T.A., Van Der Woude, L.H.V., Bergen, M.P., Stam, H.J.: Prospective study on physical activity levels after spinal cord injury during inpatient rehabilitation and the year after discharge. Assistive Technology Research Series 26, 134-136 (2010)

9. Gauthier, C., Arel, J., Brosseau, R., Hicks, A.L., Gagnon, D.H.: Reliability and minimal detectable change of a new treadmill-based progressive workload incremental test to measure cardiorespiratory fitness in manual wheelchair users. Journal of Spinal Cord Medicine 40, 759-767 (2017)

10. Van Der Scheer, J.W., De Groot, S., Tepper, M., Gobets, D., Veeger, D.J.H.E.J., Van Der Woude, L.H.V., Woldring, F., Valent, L., Slootman, H., Faber, W.: Wheelchair-specific 
fitness of inactive people with long-term spinalcord injury. Journal of Rehabilitation Medicine 47, 330-337 (2015)

11. Holloway, C., Tyler, N.: A micro-level approach to measuring the accessibility of footways for wheelchair users using the Capability Model. Transportation Planning and Technology 36, 636-649 (2013)

12. Holloway, C.S.: The effect of footway crossfall gradient on wheelchair accessibility. Department of Civil, Environmental, \& Geomatic Engineering. University College London (2011)

13. Kloosterman, M.G.M., Snoek, G.J., Van Der Woude, L.H.V., Buurke, J.H., Rietman, J.S.: A systematic review on the pros and cons of using a pushrim-activated power-assisted wheelchair. Clinical Rehabilitation 27, 299-313 (2013)

14. Cooper, R.A., Boninger, M.L., Spaeth, D.M., Ding, D., Guo, S., Koontz, A.M., Fitzgerald, S.G., Cooper, R., Kelleher, A., Collins, D.M.: Engineering Better Wheelchairs to Enhance Community Participation. IEEE Transactions on Neural Systems and Rehabilitation Engineering 14, 438-455 (2006)

15. van der Woude, L.H.V., de Groot, S., Janssen, T.W.J.: Manual Wheelchairs: Research and innovation in rehabilitation, sports, daily life, and health. Medical Engineering \& Physics 28, 905-915 (2006)

16. Consortium for Spinal Cord, M.: Preservation of upper limb function following spinal cord injury: What You Should Know (2008)

17. Holloway, C., Zuleima, D., Ramirez, M., Holloway, C.: "But, I Don't Want/Need a Power Wheelchair" : Toward Accessible Power Assistance for Manual Wheelchairs Session: Memory Impairments \& Motor Impairments 120-129 (2017)

18. Zhao, Y., Tang, J., Cao, Y., Jiao, X., Xu, M., Zhou, P., Ming, D., Qi, H.: Effects of distracting task with different mental workload on steady-state visual evoked potential based brain computer interfaces - an offline study. Frontiers in Neuroscience 12, 1-11 (2018)

19. Parasuraman, R., Sheridan, T.B., Wickens, C.D.: Situation Awareness,Mental Workload,and Trust in Automation:Viable,Empirically Supported Cognitive Engineering Constructs. Journal of Cognitive Engineering and Decision Making 2, 140-160 (2008)

20. Hancock, P.A., Parasuraman, R.: Human Factors and Safety in the Design of Intelligent Vehicle-Highway Systems. vol. 23, pp. 181-198 (1992)

21. Oyster, M.L., Smith, I.J., Kirby, R.L., Cooper, T.A., Groah, S.L., Pedersen, J.P., Boninger, M.L.: Wheelchair skill performance of manual wheelchair users with spinal cord injury. Topics in spinal cord injury rehabilitation 18, 138-139 (2012)

22. Causse, M., Chua, Z., Peysakhovich, V., Del Campo, N., Matton, N.: Mental workload and neural efficiency quantified in the prefrontal cortex using fNIRS. Scientific Reports 7, 1-15 (2017)

23. Curtin, A., Ayaz, H.: The Age of Neuroergonomics: Towards Ubiquitous and Continuous Measurement of Brain Function with fNIRS. Japanese Psychological Research (2018)

24. Ayaz, H., Dehais, F.: Neuroergonomics: The Brain at Work and in Everyday Life. Elsevier Academic Press (2018)

25. Clark, V.P., Parasuraman, R.: Neuroenhancement: Enhancing brain and mind in health and in disease. NeuroImage 85, 889-894 (2014)

26. Gramann, K., Fairclough, S.H., Zander, T.O., Ayaz, H.: Editorial: Trends in Neuroergonomics. Frontiers in Human Neuroscience 11, 11-14 (2017)

27. Karwowski, W., Siemionow, W., Gielo-Perczak, K.: Physical neuroergonomics: The human brain in control of physical work activities. Theoretical Issues in Ergonomics Science 4, 175-199 (2003)

28. Mehta, R.K., Parasuraman, R.: Neuroergonomics: a review of applications to physical and cognitive work. Frontiers in Human Neuroscience 7, 1-10 (2013)

29. Parasuraman, R.: Neuroergonomics: Research and practice. Theoretical Issues in Ergonomics Science 4, 5-20 (2003) 
30. Parasuraman, R.: Neuroergonomics: Brain, Cognition, and Performance at Work. Current Directions in Psychological Science 20, 181-186 (2011)

31. Parasuraman, R., Christensen, J., Grafton, S.: Neuroergonomics: The brain in action and at work. NeuroImage 59, 1-3 (2012)

32. Parasuraman, R., Rizzo, M.: Neuroergonomics: The brain at work. Oxford Univesity Press, New York, NY (2007)

33. Fallahi, M., Motamedzade, M., Heidarimoghadam, R., Soltanian, A.R., Miyake, S.: Assessment of operators' mental workload using physiological andsubjective measures in cement, city traffic and power plant controlcenters. Health Promotion Perspectives 6, 96$103(2016)$

34. Sauer, J., Nickel, P., Wastell, D.: Designing automation for complex work environments under different levels of stress. Applied Ergonomics 44, 119-127 (2013)

35. Chen, W.Y., Jang, Y., Wang, J.D., Huang, W.N., Chang, C.C., Mao, H.F., Wang, Y.H.: Wheelchair-related accidents: relationship with wheelchair-using behavior in active community wheelchair users. Archives of Physical Medicine and Rehabilitation 92, 892$898(2011)$

36. Roscoe, A.H.: Assessing pilot workload. Why measure heart rate, HRV and respiration? Biological Psychology 34, 259-287 (1992)

37. 2010 ADA Standards for Accessible Design. Title II 279-279 (2010)

38. Thomas, S., Reading, J., Shephard, R.J.: Revision of the Physical Activity Readiness Questionnaire (PAR-Q). Canadian journal of sport sciences $=$ Journal canadien des sciences du sport 17, 338-345 (1992)

39. https://www.slideshare.net/chessarose/wheelchair-accessible-obstacle-course

40. Meyers, A.R., Anderson, J.J., Miller, D.R., Shipp, K., Hoenig, H.: Barriers, facilitators, and access for wheelchair users: Substantive and methodologic lessons from a pilot study of environmental effects. Social Science and Medicine 55, 1435-1446 (2002)

41. Rimmer, J.H., Riley, B., Wang, E., Rauworth, A., Jurkowski, J.: Physical activity participation among persons with disabilities: Barriers and facilitators. American Journal of Preventive Medicine 26, 419-425 (2004)

42. Light, K.C., Obrist, P.A.: Task Difficulty, Heart Rate Reactivity, and Cardiovascular Responses to an Appetitive Reaction Time Task. Psychophysiology 20, 301-312 (1983)

43. Faure, V., Lobjois, R., Benguigui, N.: The effects of driving environment complexity and dual tasking on drivers' mental workload and eye blink behavior. Transportation Research Part F: Traffic Psychology and Behaviour 40, 78-90 (2016)

44. Lyu, N., Xie, L., Wu, C., Fu, Q., Deng, C.: Driver's cognitive workload and driving performance under traffic sign information exposure in complex environments: A case study of the highways in China. International Journal of Environmental Research and Public Health 14, 1-25 (2017)

45. Stikic, M., Berka, C., Levendowski, D.J., Rubio, R., Tan, V., Korszen, S., Barba, D., Wurzel, D.: Modeling temporal sequences of cognitive state changes based on a combination of EEG-engagement, EEG-workload, and heart rate metrics. Frontiers in Neuroscience 8, 1-14 (2014)

46. Hilbers, P.A., White, T.P.: Effects of wheelchair design on metabolic and heart rate responses during propulsion by persons with paraplegia. Physical Therapy 67, 1355-1358 (1987)

47. Champagne, A., Gagnon, D.H., Vincent, C.: Comparison of Cardiorespiratory Demand and Rate of Perceived Exertion During Propulsion in a Natural Environment With and Without the Use of a Mobility Assistance Dog in Manual Wheelchair Users. vol. 95, pp. 685-685. Published for the AAP by Lippincott Williams \& Wilkins, Baltimore, MD : (2016)

48. Li, W.-C., Chiu, F.-C., Kuo, Y.-s., Wu, K.-J.: The Investigation of Visual Attention and Workload by Experts and Novices in the Cockpit BT - Engineering Psychology and Cognitive Ergonomics. Applications and Services. pp. 167-176. Springer Berlin Heidelberg. 